\title{
Caracterización de la cabra criolla en la sub-provincia Volcanes de Colima y su sistema de producción
}

Macedo Barragán, R.J.; Abarca Vargas, D.; Arredondo Ruiz, V. @ y Hernandez Rivera, J.A.

Facultad de Medicina Veterinaria y Zootecnia. Universidad de Colima. México.

PALABRAS CLAVE ADICIONALES

Tipificación.

Fenotipo.

Productividad.

Socioeconomía.

Análisis multivariado.

Conservación.

\section{RESUMEN}

Se caracterizó el sistema de producción y la cabra criolla en la sub-provincia fisiográfica Volcanes de Colima. Se aplicaron 22 cuestionarios para obtener una descripción del productor, de la unidad y del sistema de producción y se realizó una caracterización faneróptica a 371 cabrasen edad reproductiva. La información se analizó usando estadística descriptiva y análisis de Componentes Principales y de Cluster Jerárquico. Las unidades de producción se orientan a la producción de carne, cuentan con una superficie y un hato promedio de 10.90 ha y 36 cabezas respectivamente. No existe manejo reproductivo, un $95 \%$ de los productores desparasita sus animales y solo el $55 \%$ los vacuna. Un $95 \%$ de las unidades productivas solo utiliza mano de obra familiar, $32 \%$ cuenta con camino de acceso asfaltado y $77 \%$ con energía eléctrica. Los productores tienen en promedio 55 años de edad, baja escolaridad, 14 años de experiencia en la cría de ganado caprino, no cuentan con acceso a financiamiento ni a asistencia. En promedio se comercializan al año 15 cabezas por productor, con un precio promedio cercano a los $\$ 33.50$ por kilogramo. En la región predominan las cabras con una capa combinada blanca y café, pelo corto y uniforme, cornamenta en forma de espiral, perfil fronto-nasal recto, orejas colgantes, mucosas negras, sin presencia de mamellas y perilla. Presentan mayoritariamente ubre de tipo globular, poco profunda, con ligamento suspensorio medio poco marcado y pezones con implantación vertical.

\section{Characterization of the creole goat in the sub-province Volcanoes of Colima and its production system}

\section{SUMMARY}

\section{ADDITIONAL KEYWORDS \\ Typification. \\ Phenotype. \\ Productivity. \\ Socioeconomics. \\ Multivariate analysis. \\ Conservation.}

\section{INFORMATION}

Cronología del artíiulo.

Recibido/Received: 05.07.2017

Aceptado/Accepted: 02.07.2019

On-line: 15.07.2019

Correspondencia a los autores/Contact e-mail:

varredondo0@ucol.mx

\section{INTRODUCCIÓN}

En México como en la mayoría de los países emergentes, la producción caprina se realiza principalmente en sistemas de producción de subsistencia, ubicados en áreas rurales de baja productividad y ligados al aprovechamiento extensivo de recursos forrajeros nativos.
A study was conducted in order to characterize the goat production system and the phenotype of creole goat in the physiographic sub-province Volcanoes of Colima. Twenty-two questionnaires including qualitative and quantitative variables related to the producer, the unit and the production system were applied and also, 371 goats in reproductive age were observed to carry out a faneroptic characterization. Data was analysed with descriptive statistics and principal component and hierarchical cluster analysis. Production units were oriented to meat production and had an average area and flock of 10.90 ha and 36 heads respectively. There is no reproductive management, $95 \%$ of producers dewormed their animals while vaccination was only implemented by $55 \%$ of them. In $95 \%$ of the farms labor was provided by the family, $32 \%$ had a paved access road and $77 \%$ electricity. Producers had an average age of 55 years, low level of schooling and 14 years of experience in goat breeding. None of the producers had access to formal credit and only $5 \%$ had access to technical assistance. On average 15 goats per producer were traded per year with an average price of $\$ 33.50 / \mathrm{kg}$. In the region prevailed goats with a combined whitebrown coat, short and uniform hair, spiral-shaped horns, straight front-nasal profile, hanging ears, black mucous membrane, without the presence of wattles and beards. They also presented to a greater extent globular-shape udders, with a poorly marked median suspensory ligament and nipples with vertical implantation.
En estas regiones, es una de las actividades de mayor importancia para la sobrevivencia de las familias campesinas, ya que la leche y principalmente la carne producida no solo satisfacen sus necesidades nutricionales básicas sino que constituye una fuente de autoempleo y de arraigo (Morand-Fehr et al. 2004; Boyazoglu et al. 2005). 
En el estado de Colima pese a existir zonas con alto potencial para el establecimiento de unidades de producción caprinas, entre ellasla sub-provincia fisiográfica Volcanes de Colima, el inventario estatal es de apenas 13,382 cabezas, con una producción anual de tan solo 116 toneladas de carne en pie y 2,800 litros de leche (INEGI 2012).

La baja productividad de estos sistemas de producción es consecuencia entre otras causas del bajo potencial productivo de las razas caprinas utilizadas. La cabra que se cría en los sistemas de producción extensivos de México es denominada "criolla", la cual se cree proviene de la raza Blanca Celtibérica o Serrana y fue introducida al continente americano por los españoles en el siglo XVI (Mellado 1997; Martínez-Rojero et al. 2004).

En este sentido, el incremento en la demanda de productos de origen animal ha obligado a mejorar la productividad de los rebaños de cabras criollas por medio de su cruzamiento con razas introducidas especializadas en la producción de leche y carne principalmente Alpina, Saanen, Toggenburg, Granadina, Nubia y Boer (Montaldo et al. 2010). Si bien con estos cruzamientos se ha logrado incrementar la producción de carne y leche (Valencia \& Montaldo 2006; Montaldo et al. 2010), existe el riesgo de la pérdida de la morfoestructura, de la rusticidad y de la adaptabilidad de las poblaciones caprinas criollas (Hernández et al. 2002; Revidatti et al. 2007).

Por otra parte, el establecimiento de programas de mejoramiento genético orientados tanto al incremento de la productividad, como a la conservación de los recursos genéticos nativos, requiere conocer las características productivas, sociales y económicas del sistema de producción bajo el cual son criados los animales (Valerio et al. 2009; Gunia et al. 2010,Sánchez Gutiérrez et al. 2018; Oliveira Moura, et al. 2019 ) así como la caracterización morfológica del animal por medio de la expresión de sus caracteres cualitativos (morfológicos y fanerópticos). Lo anterior permitirá por una parte, identificar las principales limitantes de los sistemas de producción, las tendencias de cambio en el sector ganadero, las prioridades para establecer políticas y proyectos de desarrollo, y por otro lado, establecer el estado en que se encuentra una raza en cuanto a la homogeneidad o heterogeneidad de los animales que la integran, definir su patrón racial o biotipo y determinar su aptitud y potencial productivo (Hernández et al. 2002; Revidatti et al. 2007; Herrera \& Luque, 2009).
Bajo este contexto el presente estudio tuvo como objetivo caracterizar el sistema de producción caprino y la cabra criolla en la sub-provincia fisiográfica Volcanes de Colima con vistas a su conservación.

\section{MATERIAL Y MÉTODOS}

El estudio se realizó en 22 ranchos localizados en los municipios de Colima, Comala, Cuauhtémoc y Villa de Álvarez, los cuales conforman la sub-provincia fisiográfica Volcanes de Colima. Se ubica al norte del estado, en la zona conocida como Valle de Colima y ocupa $888.50 \mathrm{~km}^{2}$ que representan el $16.03 \%$ de la superficie estatal (SIIC 2007).

Para la caracterización del sistema de producción se aplicó un cuestionario diseñado para obtener una descripción del productor, de la unidad y del sistema de producción e incluyó variables como: a) edad, sexo, escolaridad, tamaño de la familia, dependientes económicos, experiencia en la producción caprina, pertenencia a una asociación, actividades económicas alternas a la caprinocultura, acceso a fuentes de financiamiento y de asistencia técnica, b) área total, área destinada a los caprinos, tenencia de la tierra e infraestructura y c) tamaño y estructura del hato, cantidad y origen de la mano de obra, nivel de producción, mercado y manejo nutricional, sanitario, reproductivo y genético. Adicionalmente se calculó un índice técnico (animales vendidos por hembra en edad reproductiva por año). Este índice amalgama otro grupo de índices relacionados con aspectos reproductivos, sanitarios y zootécnicos como son: fertilidad, prolificidad, porcentaje de abortos, mortalidad y tasa de reemplazo (Pardos et al. 2008).

Para la caracterización faneróptica de la cabra criolla se calculó el tamaño de muestra representativa con la fórmula de Scheaffer et al. (1990), tomando como base la población caprina de los cuatro municipios a estudiar, la cual de acuerdo con el último censo agropecuario fue de 5,151 cabezas (INEGI 2012), la cual resultó en 371 animales. Posteriormente se realizó un muestreo estratificado con asignación proporcional en los cuatro municipios de acuerdo a los datos que se muestran en la Tabla I.

Esta caracterización se realizó por observación directa siguiendo la metodología deBedotti et al. (2004) evaluándose las siguientes características: color de capa, longitud de pelo, color de mucosas, tipo de cornamenta, posición de orejas, perfil fronto-nasal, pre-

Tabla I. Existencia total y proporcional de ganado caprino y animales a muestrear en los municipios de Colima, Comala, Cuauhtémoc y Villa de Alvarez, Colima (Total and proportional population of goats and animals sampled in the municipalities of Colima, Comala, Cuauhtémoc and Villa de Alvarez, Colima).

\begin{tabular}{lccc}
\hline Municipio & Existencia (cabezas) & $(\%)$ & Animales muestreados \\
\hline Colima & 2545 & 49.41 & 183 \\
Comala & 1087 & 21.10 & 78 \\
Cuauhtémoc & 787 & 15.28 & 57 \\
Villa de Álvarez & 732 & 14.21 & 53 \\
Total & 5151 & 100.00 & 371 \\
\hline${ }^{1}$ Fuente: INEGI (2012) & & &
\end{tabular}


sencia de mamellas, de perilla, de raspil, de pelliza, de arropo y de calzón, implantación del pezón, tipo y profundidad de ubrey tipo de ligamento suspensorio medio de la ubre.

Los datos fueron organizados en hojas de cálculo de Excel y para las variables de tipo cuantitativo se estimó la media, la desviación estándar y los valores mínimos y máximos mientras que en el caso de las variables de tipo cualitativo se calculó su frecuencia. Posteriormente se seleccionaron siete variables de interés en términos de clasificación, las cuales fueron además representativas de otras variables no seleccionadas y se realizó un análisis factorial de reducción de datos por el método de Componentes Principales usando la matriz rotada Varimax. Finalmente se realizó un análisis de Conglomerados Jerárquicos usando la distancia máxima o vecino más lejano como método de conglomeración y la distancia Euclídea al cuadrado como medida de intervalo para lo cual se usó el paquete estadístico SPSS v. 15.0 (SPSS 2006).

\section{RESULTADOS}

Las unidades de producción tienen una superficie promedio de 10.90 ha de las cuales solo un $52 \%$ se utiliza para la producción de caprinos y el resto se destina a otras actividades agrícolas y ganaderas. El tamaño promedio del hato es de 36 cabezas, 62\% de las cuales conforman el pie de cría (hembras en edad reproductiva) y en promedio las unidades de producción cuentan con un semental, destacando la presencia de una unidad con tres sementales y otra que no cuenta con un reproductor.

Únicamente un $46 \%$ de las unidades de producción combina la cría de caprinos con la agricultura y la cría de otra especie pecuaria, un $36 \%$ de ellas combina la cría de caprinos con otra especie pecuaria y solo un $18 \%$ se dedica a la cría exclusiva de caprinos. Entre los cultivos establecidos se destaca el maíz y entre las especies animales presentes se encuentran principalmente ovinos, bovinos y aves de corral. Todas las unidades de productivas se orientan a la producción de carne, un $64 \%$ de ellas lo realiza bajo un sistema de producción semi-intensivo, un $18 \%$ bajo un sistema extensivo y en un $18 \%$ se tiene establecido un sistema de producción intensivo. El pastoreo de la vegetación nativa compuesta mayoritariamente por huizache (Acacia farnesiana), guazima (Guazumaulmifolia), guamuchil (Pithecellobium dulce), mezquite (Prosopisjuliflora), espino blanco (Acacia acatlensis) y gramas así como de algunos pastos introducidos principalmente estrella africana (Cynodonplectostachyus) constituye la base de la alimentación en la mayoría de las explotaciones, el cual es complementado con grano de maíz y sorgo, rastrojo de maíz, punta de caña y alimento comercial para cerdos y ganado lechero.

No existe manejo reproductivo en los hatos, predominando el empadre de tipo continuo (95\%). En la mayoría de las explotaciones el píe de cría se compone por una mezcla de hembras con diversos patrones raciales, en su mayoría indefinido, mientras que en el caso de los machos, un 59\% de las unidades de producción cuenta con sementales de la raza Boer, un 18\% con
Tabla II. Precio de venta, índice técnico, producción anual vendida y para autoconsumo de las unidades de producción en la sub-provincia Volcanes de Colima (Sales price, technical index, sold and self-consumption annual production of the production units in the sub-province Volcanoes of Colima).

\begin{tabular}{lcccc}
\hline Variable & Media & D.E & Mínimo & Máximo \\
\hline $\begin{array}{l}\text { Animales totales } \\
\text { vendidos (cabezas/año) }\end{array}$ & 15.00 & 14.37 & 0.00 & 45.00 \\
$\begin{array}{l}\text { Animales autoconsumo } \\
\text { (cabezas/año) }\end{array}$ & 2.55 & 3.33 & 0.00 & 14.00 \\
$\begin{array}{l}\text { Precio de venta machos } \\
(\$ / \mathrm{kg})^{1}\end{array}$ & 34.84 & 4.55 & 25.00 & 40.00 \\
$\begin{array}{l}\text { Precio venta hembras }(\$ / \\
\mathrm{kg})^{1}\end{array}$ & 32.50 & 4.24 & 25.00 & 40.00 \\
Índice técnico $^{2}$ & 0.67 & 0.61 & 0.00 & 2.50 \\
\hline
\end{tabular}

${ }^{1}$ Pesos mexicanos. 1 Dólar americano $=19.00$

${ }^{2}$ Animales vendidos por hembra en edad reproductiva por año.

sementales de esta raza cruzados con razas criollas, un $18 \%$ con sementales criollos y una sola de ellas (5\%) cuenta con ejemplares de la raza Nubia.

Por lo que respecta al manejo sanitario, si bien la desparasitación de los animales es una práctica sanitaria ampliamente extendida dentro los productores (95\%), la vacunación solo es realizada por un 55\% de ellos. Entre las enfermedades de mayor ocurrencia en los hatos se encontraron la pododermatitis infecciosa y diversas infecciones del aparato respiratorio y del aparato digestivo.

En un 95\% de las unidades de producción solo se utiliza mano de obra familiar, un $32 \%$ de ellas cuenta con camino de acceso asfaltado mientras que una amplia mayoría (77\%) cuenta con energía eléctrica y cuentan con un régimen de tenencia de la tierra de pequeña propiedad (82\%). En la mayoría de las explotaciones la infraestructura se limita a un corral techado para el encierro nocturno de los animales equipado con comederos y bebederos, todo esto construido con frecuencia con materiales de desecho o de reúso.

La edad promedio del productor fue de 55 años, con una familia integrada por cuatro personas en promedio. Su escolaridad es baja con apenas educación básica concluida (6 años de estudio) y su experiencia en la cría de ganado caprino se puede considerar amplia con más de 14 años en promedio dedicados a la actividad.

Solo la mitad de los productores vive en la unidad de producción, la totalidad de ellos no cuenta con acceso a una fuente de financiamiento, 95\% no recibe asistencia técnica y solo un $14 \%$ pertenece a alguna asociación de productores. Un 91\% de los productores manifestó contar con otra actividad económica distinta a la caprinocultura, destacando la agricultura (32\%), la ganadería (23\%) y el comercio (18\%), de ellos un $59 \%$ manifestó que la cría de caprinos representa su principal fuente de ingresos.

En promedio se comercializan solo 15 cabezas por año de los cuales una tercera parte son hembras, con un precio promedio de $\$ 33.50 / \mathrm{kg}$, en tanto que en este mismo periodo de tiempo los productores destinan 


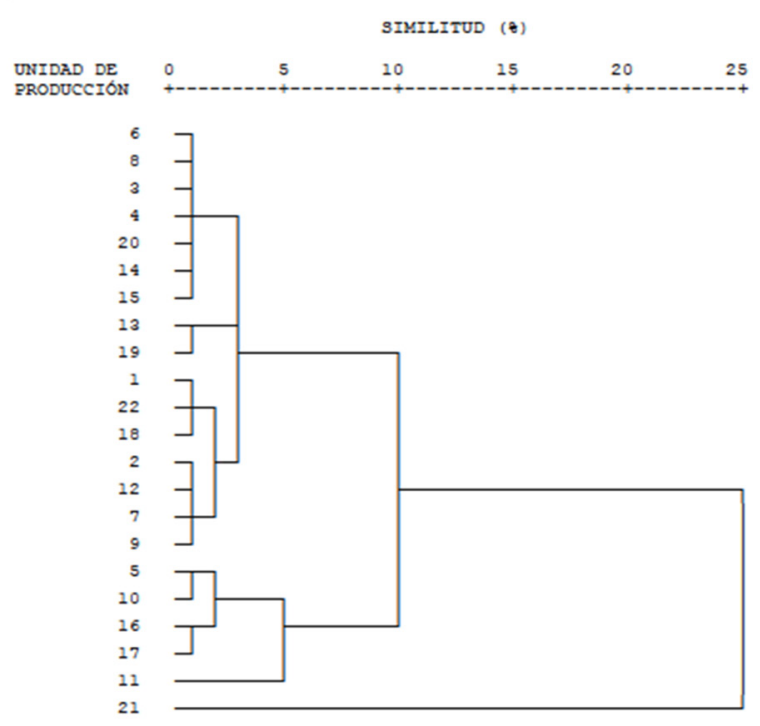

Figura 1. Dendrograma de clasificación por similitud de las unidades de producción caprina de la subprovincia Volcanes de Colima (Dendrogram of classification by similarity of the goat production units of the sub-province Volcanoes of Colima).

solo 2.5 animales en promedio a autoconsumo. Asimismo, el índice técnico indica que en promedio se comercializa poco más de media cabeza por hembra en etapa reproductiva por año (Tabla II).

El análisis factorial generó seis componentes principales, tres de los cuales explicaron el $94.30 \%$ de la variación total. El componente 1 explicó el $74.20 \%$ de la varianza y es caracterizado por la variable tamaño del hato. Los componentes 2 y 3 explicaron el 12.50 y el $7.70 \%$ de la variación y son caracterizados por la edad y la experiencia del productor en la cría de cabras respectivamente (Tabla III).

El análisis de Conglomerados Jerárquicos mostró una alta homogeneidad entre las unidades de producción y los productores, formándose dos grupos bien definidos, el primero (Grupo 1) formado por dieciséis unidades de producción con un nivel de similitud del 97\%, y el segundo (Grupo 2) formado por cinco unidades con una similitud del 95\%. Una unidad de producción se mantuvo independiente agrupándose con los dos grupos de unidades de producción a un nivel de similitud del 75\% (Figura 1).

El Grupo 1 incluyó las 16 unidades de producción con la menor superficie y tamaño de hato. Los productores son de mediana edad sin educación primaria terminada y presentan la menor experiencia en la producción de cabras. El Grupo 2 se encuentra constituido por cinco unidades productivas con superficie y hatos de tamaño intermedio. Los productores son los más jóvenes de la región, cuentan con estudios de educación elemental concluidos y una experiencia intermedia en la caprinocultura. El Grupo 3 está conformado por una sola unidad que posee la mayor superficie y hato. El productor tiene estudios concluidos de educación secundaria y es el de mayor edad y experiencia en la actividad (Tabla IV).

En la región de estudio y en los municipios de Colima, Cuauhtémoc y Villa de Álvarez predominaron las cabras con una capa combinada blanca y café, mientras que de manera particular en el municipio de Comala predominaron las cabras con color de capa café (Tabla V). El tipo de pelo predominante fue corto y uniforme aunque en las cabras del municipio de Cuauhtémoc se observó un predominio de pelo de tamaño medio y en las del municipio de Colima se observó mayoritariamente la presencia de raspil (Tabla VI).

La mayoría de las cabras no presentaron mamellas y perilla (Tabla VII), en tanto se observó una mayor frecuencia de cabras con cornamenta en forma de espiral, con perfil fronto-nasal recto, con orejas colgantes y con mucosas negras. De forma particular las cabras del municipio de Cuauhtémoc presentaron cuernos en forma de arco y mucosas de coloración rosada, en tanto que en las del municipio de Comala se observó la presencia de mucosas pigmentadas (Tabla VIII).

Las cabras de la zona estudiada mostraron de manera predominante una ubre de tipo globular, poco profunda, con un ligamento suspensorio medio poco marcado y con pezones de implantación vertical. En el caso de las cabras del municipio de Cuauhtémoc

Tabla III. Estructura factorial de las unidades de producción en la sub-provincia Volcanes de Colima (Structure matrix of the production units in the sub-province Volcanoes of Colima).

\begin{tabular}{|c|c|c|c|c|c|}
\hline \multirow[b]{2}{*}{ Variable } & \multicolumn{5}{|c|}{ Componente principal } \\
\hline & 1 & 2 & 3 & 4 & 5 \\
\hline Área total & 0.3639 & -0.5457 & -0.3795 & 0.6446 & -0.1015 \\
\hline Tamaño del hato & 0.9175 & 0.2494 & -0.0109 & -0.3034 & 0.0618 \\
\hline Edad del productor & 0.0147 & -0.7706 & 0.1058 & -0.6168 & -0.1186 \\
\hline Escolaridad & 0.0194 & 0.1638 & 0.0317 & -0.0083 & -0.9853 \\
\hline Experiencia & 0.1588 & -0.1394 & 0.9184 & 0.3338 & 0.0062 \\
\hline Índice técnico & 0.0010 & -0.0009 & -0.0112 & -0.0220 & -0.0295 \\
\hline Precio de venta & 0.0014 & 0.0035 & -0.0033 & -0.0086 & -0.0054 \\
\hline Valor propio & 1214.31 & 203.72 & 125.34 & 88.28 & 4.17 \\
\hline Variación explicada (\%) & 74.20 & 12.50 & 7.70 & 5.40 & 0.30 \\
\hline Variación acumulada (\%) & 74.20 & 86.70 & 94.30 & 99.70 & 100.00 \\
\hline
\end{tabular}


se observó la presencia mayoritaria de ubres de tipo piriforme (Tabla IX).

\section{DISCUSIÓN}

La superficie de las unidades de producción, al igual que las ubicadas en el estado de Puebla, México fue menor a 10 hectáreas, de las cuales de una tercera a cuarta parte se destina al cultivo del maíz. Asimismo, en la explotación se crían otras especies animales como aves, cerdos ovinos y bovinos sin la existencia de una verdadera especialización en alguna de las actividades (Serrano 2010; Trujillo 2012). La extensión de las unidades productivas mexicanas contrasta con las observadas en otros países de Latinoamérica como República Dominicana y Colombia, en donde las estas superan las 170 hectáreas (Valerio et al. 2009; Atuesta et al., 2012; Vargas et al., 2014).

El tamañodel hato fue similar al encontrado por diversos autores en varias regiones del país como la región de Libres, Puebla(Serrano 2010), la Cordillera del Tzenzo, Puebla (Vargas 2003) y la región de Tlalixtaquilla, Guerrero (Sedano 2012), en las cuales la mayoría de los hatos están compuestos por 20 a 40 animales. Sin embargo fue significativamente menor a los encontrados en Coahuila y en el sistema transhumante de la Mixteca oaxaqueña, los cuales se conforman por 150 a 500 y 900 a 1500 cabezas respectivamente (Ruiz et al. 1992; Ramírez et al. 2014). Es importante señalar que prácticamente todos los rebaños estudiados cuentan con al menos un semental mientras que en algunas zonas de Puebla solo el $66.66 \%$ de los hatos cuentan con semental y en aquellos rebaños integrados por menos de 26 animales, estos son rentados o prestados (Serrano 2010). Asimismo, al igual que en el estado de Puebla, se observó una tendencia hacia la introducción de sementales de la raza Boer (Silva-Gómez et al. 2015).

Es de notar que el 100\% de las unidades de producción estudiadas están orientadas a la producción de carne, situación explicada por el bajo consumo de leche caprina y sus derivados en el estado de Colima, lo que coincide con los estudios realizados en el sur del estado de México (Dorantes et al. 2012), en el estado de Puebla (Hernández et al. 2012) y en Mixteca oaxaqueña (Ramí-

\begin{tabular}{|c|c|c|c|}
\hline \multirow[b]{3}{*}{ Variable } & \multirow{2}{*}{\multicolumn{3}{|c|}{ Grupo }} \\
\hline & & & \\
\hline & 1 & 2 & 3 \\
\hline Tamaño del hato (cabezas) & 21.13 & 63.00 & 150.00 \\
\hline Área total (ha) & 4.19 & 23.22 & 50.00 \\
\hline Experiencia & 10.93 & 23.00 & 25.00 \\
\hline Edad (años) & 55.44 & 52.40 & 59.00 \\
\hline Escolaridad (años) & 5.63 & 6.60 & 9.00 \\
\hline Precio de venta $(\$)^{1}$ & 34.80 & 35.00 & 40.00 \\
\hline Índice técnico ${ }^{2}$ & 0.65 & 0.74 & 0.57 \\
\hline
\end{tabular}

${ }^{1}$ Pesos mexicanos. 1 Dólar americano $=19.00$

${ }^{2}$ Animales vendidos por hembra en edad reproductiva por año rez et al. 2014). Lo anterior contrasta con los sistemas productivos de la Comarca Lagunera en México y de numerosas regiones de España, los cuales en su mayoría están orientados a la producción de leche (Castel et al. 2010; Escareño et al. 2011). De la misma forma, se coincide con una gran cantidad de trabajos realizados en diversos países que indican que el pastoreo de la vegetación nativa constituye la principal fuente de alimentación de las cabras (Serrano 2010; Guerrero et al. 2012; Vargas et al. 2014) y tal y como lo mencionan Serrano (2010) y Trujillo (2012), en México el rastrojo y grano de maíz constituyen la principal fuente de complementación alimenticia de los hatos.

En lo referente al manejo sanitario que los productores realizan en sus hatos, destaca que el 95\% de los productores desparasitan sus animales dos veces al año, proporción similar a la registrada en la región de Libres, Puebla (Serrano 2010) y en el sur del estado de México (Dorantes et al. 2012). Igualmente, se concuerda con las observaciones realizadas en Puebla, sobre la falta de un programa de vacunación en sus rebaños, a pesar de que en aquella entidad las enfermedades neumónicas representan una de las principales causas de mortalidad en sus hatos y solo aceptan vacunar a sus cabras contra la brucelosis por disposición oficial durante la temporada de campaña (Serrano 2010). La falta de manejo reproductivo en los hatos, caracterizada como la práctica del empadre continuo, es similar a la observada por Escareño et al. (2011) y por Ramírez et al. (2014) en la Comarca Lagunera y en la Mixteca oaxaqueña, en donde durante todo el año los machos permanecen junto a las hembras.

Al igual que en otras regiones del país, los productores de la zona estudiada mostraron una edad mayor a los 50 años y una baja escolaridad (Dorantes et al. 2012; Trujillo 2012) y aunque en promedio cuentan con 14 años de experiencia en la cría de cabras, esta es

Tabla V. Frecuencia de color de capa de la cabra criolla de la sub-provincia Volcanes de Colima (Frequency of coat color of the creole goat of the sub-province Volcanoes of Colima).

\begin{tabular}{|c|c|c|c|c|c|}
\hline \multicolumn{6}{|l|}{ Municipio(1) } \\
\hline Característica & 1 & 2 & 3 & 4 & Sub-provincia \\
\hline \multicolumn{6}{|l|}{ Color de capa } \\
\hline Negra & 0.00 & 7.65 & 3.51 & 5.67 & 5.12 \\
\hline Blanca & 23.08 & 14.21 & 19.30 & 15.09 & 16.98 \\
\hline Colorada & 5.13 & 15.85 & 8.77 & 1.88 & 10.51 \\
\hline Café & 37.18 & 1.64 & 22.81 & 13.21 & 14.02 \\
\hline Blanca + Café(2) & 14.10 & 23.50 & 24.57 & 32.09 & 22.92 \\
\hline Blanca + Negra(2) & 1.28 & 10.93 & 1.75 & 7.55 & 7.01 \\
\hline Café + Blanco ${ }^{(2)}$ & 8.97 & 6.01 & 5.26 & 9.43 & 7.01 \\
\hline Café + Negra(2) & 5.13 & 5.46 & 5.26 & 9.43 & 5.93 \\
\hline Negra + Blanca ${ }^{(2)}$ & 1.28 & 5.46 & 0.00 & 3.77 & 3.50 \\
\hline Negra + Café(2) & 3.85 & 4.37 & 0.00 & 1.88 & 3.23 \\
\hline Pinta $^{(3)}$ & 0.00 & 4.92 & 8.77 & 0.00 & 3.77 \\
\hline
\end{tabular}

11 = Comala, 2 = Colima, $3=$ Cuauhtémoc, 4 = Villa de Álvarez

${ }^{2}$ El primer color es predominante

${ }^{3}$ Presenta una combinación de más de dos colores. 
Tabla VI. Frecuencia de la longitud y el tipo de pelo de la cabra criolla de la sub-provincia Volcanes de Colima (Frequency of length and type of hair of the creole goat of the sub-province Volcanoes of Colima).

\begin{tabular}{lccccc}
\hline Municipio(1) & & & & & \\
\hline Característica & 1 & 2 & 3 & 4 & 5 \\
\hline Longitud de pelo & & & & & \\
Corto & 85.90 & 56.84 & 31.58 & 64.16 & 60.11 \\
Medio & 12.82 & 37.70 & 63.16 & 33.96 & 35.85 \\
Largo & 1.28 & 5.46 & 5.26 & 1.88 & 4.04 \\
Distribución de pelo & & & & & \\
Uniforme & 41.04 & 30.60 & 57.89 & 45.28 & 39.07 \\
Raspil & 39.74 & 45.35 & 24.57 & 16.98 & 36.93 \\
Pelliza & 2.56 & 0.00 & 0.00 & 0.00 & 0.54 \\
Calzón & 0.00 & 0.55 & 0.00 & 5.67 & 1.08 \\
Raspil + Calzón & 12.82 & 18.03 & 14.04 & 26.42 & 17.52 \\
Raspil + Calzón + Arropo & 0.00 & 1.09 & 0.00 & 0.00 & 0.54 \\
Raspil + Calzón + Pelliza & 2.56 & 2.19 & 1.75 & 1.88 & 2.16 \\
$\begin{array}{l}\text { Pelliza + Calzón + Arropo } \\
\text { Raspil + Calzón + Pelliza }\end{array}$ & 0.00 & 0.55 & 0.00 & 0.00 & 0.27 \\
+ +Arropo & 1.28 & 1.64 & 1.75 & 3.77 & 1.89 \\
\hline $\begin{array}{l}\text { 11 = Comala, 2 = Colima, 3 = Cuauhtémoc, } 4=\text { Villa de Álvarez, } \\
\text { 5= Sub-provincia. }\end{array}$ & & & & & \\
\hline
\end{tabular}

significativamente menor a la de los productores de la Comarca Lagunera y dela Mixteca Poblana, los cuales tienenen promedio 19 y 27 años dentro de la actividad respectivamente (Escareño et al. 2011; Trujillo 2012).En este sentido varios estudios muestran una relación positiva entre la escolaridad, la edad y la experiencia de los productores con la eficiencia técnica de las unidades de producción caprina (Gül et al. 2016).

Como se observó en el presente estudio, los caprinocultores cuentan con familias numerosas cuyos integrantes constituyen la principal fuente de mano de obra en las unidades de producción. Tal y como sucede en otras regiones de México y en varios países en desarrollo, la mano de obra familiar es fundamental para la subsistencia de los sistemas de producción caprinos, ya que contribuye a su supervivencia y a mejorar su rentabilidadpermitiendo además,la incorporación de sectores de la sociedad socialmente vulnerables como mujeres, niños y ancianosal manejo de los animales y al procesamiento de sus derivados (Valerio et al. 2009; Hulela 2010; Vázquez-García2015).

Un aspecto negativo observado entre los productores fue su falta de acceso a programas de asistencia técnica, de financiamiento y de pertenencia a algún tipo de asociación u organización de productores. La pertenencia a una asociación legalmente constituida ha permitido a los caprinocultores de otras zonas del país recibir apoyo económico para la adquisición de infraestructura y de animales por parte del sector gubernamental,recibir asistencia técnica por parte de instituciones educativas y favorecer el proceso de transferencia de tecnología entre productores organizados (Vargas 2003; Serrano 2010).Recientemente, autores como Gül et al. (2016)
Tabla VII. Presencia de mamellas y de perilla en la cabra criolla de la sub-provincia fisiográfica Volcanes de Colima (Presence of wattles and beard in the creole goat of the sub-province Volcanoes of Colima).

\begin{tabular}{|c|c|c|c|c|c|}
\hline \multicolumn{6}{|l|}{ Municipio(1) } \\
\hline Característica & 1 & 2 & 3 & 4 & Sub-provincia \\
\hline \multicolumn{6}{|c|}{ Presencia de mamellas } \\
\hline No & 96.15 & 95.08 & 87.72 & 98.12 & 94.61 \\
\hline $\mathrm{Si}$ & 3.85 & 4.92 & 12.28 & 1.88 & 5.39 \\
\hline \multicolumn{6}{|c|}{ Presencia de perilla } \\
\hline No & 57.69 & 74.32 & 56.14 & 58.49 & 65.77 \\
\hline $\mathrm{Si}$ & 42.31 & 25.68 & 43.86 & 41.51 & 34.23 \\
\hline
\end{tabular}

y Gaspar et al. (2016)concluyen que la integración de productores de pequeños rumiantes en estructuras cooperativas y asociaciones puede incrementar su eficiencia técnica, mejorar las unidades de producción y asegurar la persistencia de las mismas gracias a la provisión de servicios adicionales al ganadero, a la generación de valor añadido y a la estabilidad en los precios percibidos.

Aunque más del $90 \%$ de los productores manifestaron que cuentan con una fuente de ingresos distinta a la cría de cabras, casi la mitad indicó que esta actividad representaba su principal fuente de ingresos, situación similar a la observada en Libres, Puebla (Serrano, 2010). La proporción de productores que complementan su ingreso con actividades realizadas al exterior de

Tabla VIII. Frecuencia de tipo de cornamenta, perfil fronto-nasal, posición de orejas y color de mucosas de la cabra criolla de la sub-provincia Volcanes de Colima (Frequency of antler type, fronto-nasal profile, ear position and mucous color of the creole goat of the sub-province Volcanoes of Colima).

\begin{tabular}{|c|c|c|c|c|c|}
\hline \multicolumn{6}{|l|}{ Municipio(1) $^{(1)}$} \\
\hline Característica & 1 & 2 & 3 & 4 & Sub-provincia \\
\hline \multicolumn{6}{|c|}{ Tipo de cornamenta } \\
\hline Acorne & 26.92 & 14.75 & 17.54 & 16.98 & 18.06 \\
\hline Arco & 24.36 & 24.04 & 57.89 & 26.42 & 29.65 \\
\hline Espiral & 48.72 & 61.21 & 24.57 & 56.60 & 52.29 \\
\hline \multicolumn{6}{|c|}{ Perfil fronto-nasal } \\
\hline Cóncavo & 0.00 & 0.00 & 1.75 & 0.00 & 0.27 \\
\hline Recto & 51.28 & 63.39 & 52.64 & 54.72 & 57.95 \\
\hline Convexo & 48.72 & 36.61 & 45.61 & 45.28 & 41.78 \\
\hline \multicolumn{6}{|c|}{ Posición de orejas } \\
\hline Verticales & 0.00 & 0.00 & 0.00 & 0.00 & 0.00 \\
\hline Horizontales & 39.74 & 24.59 & 29.82 & 32.08 & 29.65 \\
\hline Colgantes & 60.26 & 75.41 & 70.18 & 67.92 & 70.35 \\
\hline \multicolumn{6}{|c|}{ Color de mucosas } \\
\hline Negra & 33.33 & 60.10 & 19.30 & 45.28 & 46.09 \\
\hline Rosada & 8.97 & 11.48 & 63.16 & 41.51 & 23.18 \\
\hline Pigmentada & 57.70 & 28.42 & 17.54 & 13.21 & 30.73 \\
\hline
\end{tabular}


Tabla IX. Frecuencia de tipo de ubre y de ligamento, de profundidad de la ubre y de implantación del pezón en la cabra criolla de la sub-provincia Volcanes de Colima (Type of udder and ligament, depth of the udder and implantation of nipple in the creole goat of the subprovince Volcanoes of Colima).

\begin{tabular}{|c|c|c|c|c|c|}
\hline \multicolumn{6}{|l|}{ Municipio(1) } \\
\hline Característica & 1 & 2 & 3 & 4 & 5 \\
\hline \multicolumn{6}{|l|}{ Tipo de ubre } \\
\hline Oval & 21.79 & 20.77 & 28.07 & 37.74 & 24.53 \\
\hline Globular & 50.00 & 44.26 & 21.05 & 47.17 & 42.32 \\
\hline Piriforme & 28.21 & 34.97 & 50.88 & 15.09 & 33.15 \\
\hline \multicolumn{6}{|l|}{ Profundidad de la ubre } \\
\hline Poco profunda & 74.36 & 86.34 & 71.92 & 94.34 & 82.75 \\
\hline $\begin{array}{l}\text { Moderadamente } \\
\text { profunda }\end{array}$ & 23.08 & 10.93 & 24.57 & 5.66 & 14.82 \\
\hline Muy profunda & 2.56 & 2.73 & 3.51 & 0.00 & 2.43 \\
\hline \multicolumn{6}{|c|}{ Tipo de ligamento suspensorio medio } \\
\hline Poco marcado & 50.00 & 55.75 & 57.89 & 54.72 & 54.72 \\
\hline Moderadamente marcado & 10.26 & 11.48 & 14.04 & 30.19 & 14.29 \\
\hline Muy marcado & 39.74 & 32.79 & 28.07 & 15.09 & 30.99 \\
\hline \multicolumn{6}{|l|}{ Implantación del pezón } \\
\hline Lateral & 11.54 & 16.94 & 42.11 & 37.74 & 22.64 \\
\hline Vertical & 84.61 & 82.51 & 56.14 & 60.38 & 75.74 \\
\hline
\end{tabular}

11 = Comala, 2 = Colima, 3 = Cuauhtémoc, 4 = Villa de Álvarez, $5=$ Sub-provincia.

la unidad de producción (18\%) también fue similar a la observada en el mencionado estudio.Con respecto a la comercialización, mientras que en el presente estudio se venden anualmente en promedio 15 animales por productor, obtenidos de un hato promedio de 37 animales, en algunas unidades de producción del estado de México, se tienen hatos conformados por 36 animales en promedio de los cuales se comercializan anualmente 46 animales (Dorantes, 2013). El precio de venta registrado entre los productores estudiados fue menor al indicado por Silva-Gómez et al. (2015) en el estado de Puebla, en donde en el año 2015el precio para los machos y hembras criollas con un peso de entre 45 a $50 \mathrm{~kg}$ y 30 a $35 \mathrm{~kg}$ respectivamente ya se ubicaba en $\$ 40.00 / \mathrm{kg}$. Cabe señalar que en este estudio, los animales consumidos por el productor representaron un $17 \%$ de los comercializados, mientras que en el sur del estado de México los animales para autoconsumo llegan a representar el $27 \%$ de los animales producidos (Dorantes et al., 2012) y en el estado de Puebla este fue mínimo (Hernández et al., 2012).

De acuerdo con Mellado (1997), las cabras criollas de México derivan las cabras traídas por los españoles al continente americano en el siglo XVI, principalmente de las razas Blancas Celtibérica o Serrana y Castellana de Extremadura. La cabra Blanca Celtibérica presenta en general un color de capa blanco mate (Luque, 2011) mientras que la cabra Castellana actualmente denominada Verata, posee un color de capa castaño (Rodríguez et al., 1990). En este sentido, la influencia de estas dos razas sigue de manifiesto en las poblaciones criollas de cabras como las estudiadas en este trabajo, entre las cuales los colores de capa predominantes son blanco, café y sus distintas combinaciones. Existen otras poblaciones caprinas en los estados de Guerrero y Zacatecas, en las cuales predomina el color blanco y crema (Reveles et al. 2008; Martínez et al. 2013), característica que de acuerdo con Agraz (1989) es afín a las de sus predecesoras, las cabras Celtibéricas.

El perfil fronto-nasal y el tipo de cornamenta constituyen dos características de máximo interés para definir los patrones raciales de los caprinos (Aparicio 1960). En el presente estudio predominaron las cabras con cuernos en forma de espiral y con perfil frontonasal recto, lo que difiere de otros trabajos realizados en México en los cuales se encontró una predominancia de cabras con cuernos en forma de arco (De los Santos 2011; Sedano 2012). Estudios previos indican que en algunas de las razas españolas que dieron origen a las poblaciones de cabras criollas en América como laBlanca de Rasquera, la Blanca Celtibérica, la Blanca Andaluza y laVerata(Rodríguez et al. 1990; Luque et al. 2005; Carne et al. 2007) predomina la cornamenta en espiral y en la raza Blanca Celtibérica (Luque 2011) el perfil fronto-nasal recto,lo que explica los hallazgos del presente estudio.

El predominio de cabras con orejas colgantes ubica a las cabras de la región estudiada como de tipo Nubiano. Este rasgo en conjunto con un perfil fronto-nasal convexo y cornamenta en forma de arco, sugiere un alto grado de cruzamiento con sementales de la raza Nubia y de la raza Boer, situación también observada en otros hatos del país, en los cuales se introdujeron estas dos razas desde Europa y los Estados Unidos de América respectivamente para mejorar la producción de carne en los sistemas de producción de tipo extensivo (Montaldo et al. 2010; Martínez et al. 2013). Lo anterior difiere de lo observado en otras poblaciones de cabras ubicadas en México que presentan en su mayoría orejas erectas y horizontales (Hernández et al. 2002; Sedano 2012; Martínez et al. 2013). De acuerdo con Nozawa (1991).

La forma, estructura y distribución de los filamentos pilosos llegan a constituir caracteres étnicos esenciales para la clasificación de las diferentes poblaciones de caprinos (Aparicio 1960). Aunque en este trabajo predominaron las cabras con un pelaje uniforme, un porcentaje importante de estas mostraron la presencia de raspil. Cabe mencionar que en la cabra Blanca Celtibérica una de las razas que dio origen a las cabras criollas mexicanas es frecuente la presencia de raspil y de pelliza (Ministerio de Agricultura y Pesca, Alimentación y Medio Ambiente, 2017).

La presencia de mamellas, es un carácter que al parecer no tiene importancia en la definición del tipo racial pero que ha sido relacionado con aspectos reproductivos. De acuerdo con Ricordeau (1967), las cabras que presentan el gen responsable de la presencia de mamellas dominante ya sea en forma homo o heterocigótica son un $13 \%$ más prolíficas que aquellas que lo presentan en forma recesiva y homocigótica. Asimismo, las cabras con mamellas y ausencia de cuernos son $25.6 \%$ más prolíficas que el promedio. En el presente estudio la proporción de individuos con mamellas fue 
inferior al encontrado por Sedano (2012) en cabras Pastoreñas en Guerrero y por Martínez et al. (2013) en rebaños de cabra Blanca de "Filo Mayor" en Guerrero en donde un $53.70 \%$ de individuos presentaban estos apéndices.Por lo que respecta a la presencia de perilla, un $42.31 \%$ de las cabras estudiadas la presentaron, lo cual resulta superior a lo encontrado por Sedano (2012) quien indicó que esta solo estuvo presente en un $20 \%$ de las cabras Pastoreñas.

En el presente trabajo predominaron las cabras con ubres de tipo globular, coincidiendo con otros estudios realizados en poblaciones de cabras criollas en la Sierra Madre del Sur en el estado de Guerrero (Martínez et al. 2013) y en el estado de Puebla (Hernández et al. 2002; Serrano 2010. Las cabras con este tipo de ubre son preferidas para el pastoreo, por ser ubres recogidas hacia la cavidad abdominal y por lo tanto menor susceptibilidad a sufrir heridas o traumatismos provocados por el golpeteo con los matorrales (Martínez et al. 2013). Es importante señalar que una gran mayoría de las cabras estudiadas mostraron pezones con implantación vertical. La colocación de los pezones es un carácter determinante para la facilidad de ordeño ya que estos deben estar colocados justo debajo de cada glándula para facilitar el ordeño por gravedad. La implantación del pezón se encuentra además estrechamente correlacionada con la inclinación y orientación de los mismos, de tal forma que unos pezones implantados en su sitio generalmente permiten un buen ordeño, incluso aunque su inclinación y orientación no sean las más correctas (Ruiz 2008; Sánchez et al. 2009).

El ligamento suspensorio medio es el soporte principal de la ubre y por lo tanto debe ser fuerte y capaz de mantener en su sitio ubres de gran producción durante mucho tiempo. Existen ubres con suelo redondo sin ligamento medio funcional, y otras con un ligamento excesivamente fuerte que parte en dos la ubre, lo que hace que pierda capacidad, por lo que es deseable la presencia de ligamentos de tipo intermedio (Ruiz 2008; Sánchez et al. 2009), los cuales solo fueron observados en una de cada siete cabras estudiadas.

La profundidad de la ubre se valora observando la distancia que existe entre el piso de la ubre y los corvejones. Así, se pueden encontrar ubres muy profundas, con muchos centímetros por debajo de los corvejones, hasta ubres muy altas, bastantes centímetros por encima de estos. Las ubres profundas no son deseables ya que son más propensas a sufrir lesiones, especialmente en el periparto y dificultan además el ordeño mecánico. Por otro lado las ubres muy altas tienen poca capacidad, de modo que lo deseable son ubres intermedias, con el suelo de la ubre aproximadamente a la altura de las puntas de los corvejones. Estas además, se encuentran bastante protegidas, facilitan el ordeño, tienen suficiente capacidad y soportan lactaciones sucesivas sin descolgarse (Ruiz 2008; Sánchez et al. 2009). En el presente trabajo un $82.75 \%$ de las ubres mostraron poca profundidad, lo que denota un bajo potencial productivo de las cabras de la región en estudio.

Se concluye por una parte, que la producción caprina en la sub-provincia fisiográfica Volcanes de Colima es una actividad orientada a la producción de carne con baja productividad, practicada en unidades de producción de poca extensión y con poco uso de tecnología, cuyo desarrollo deberá incluir principalmente la incorporación de productores jóvenes con acceso a asistencia técnica y a fuentes de financiamiento. Por otra lado, la cabra criolla de la sub-provincia fisiográfica Volcanes de Colima presenta una gran variabilidad fenotípica y si bien algunas características predominantes como el color de capa, el perfil fronto-nasal y el tipo de cornamenta guardan aún consistencia con las razas que ingresaron a México durante la conquista, el presente estudio evidenció la formación de nuevos fenotipos, producto de la hibridación con otras razas de reciente introducción principalmente Boer.

\section{AGRADECIMIENTOS}

Los autores agradecen a la Unión Ganadera Regional de Colima por el patrocinio para el desarrollo del proyecto "Estudio de la cabra criolla en la provincia eje neovolcánico del estado de Colima".

\section{BIBLIOGRAFÍA}

Agraz, GAA1989, Caprinotecnia II, Limusa, México, D.F.

Aparicio, G 1960,Zootecnia Especial. Etnología compendiada, Imprenta Moderna, Córdoba, España.

Atuesta, MF, Daza, LM, Del Rio, FA, Garnica, YM, Martínez, DA, Serrano, NCA, Vega, JL\& Vargas, BJ2012,'Caracterización de los sistemas productivos caprinos en el municipio de Villanueva, Santander', Actas Iberoamericanas de Conservación Animal, vol. 2, pp. 293-96.

Bedotti, D, Gómez-Castro, AG, Sánchez-Rodríguez, M\&Martos-Peinado, J2004,'Caracterización morfológica y faneróptica de la cabra colorada pampeana',Archivos de Zootecnia,vol. 53, pp.261-271.

Boyazoglu, J, Hatziminaoglou, I \&Morand-Fehr, P2005,'The role of the goat in society: past, present and perspectives for the future', Small Ruminant Research, vol. 60, pp.13-23.

Castel, JM, Ruiz, FA, Mena, Y \&Sánchez-Rodríguez, M2010, 'Present situation and future perspectives for goat production systems in Spain',Small Ruminant Research,vol.89, pp. 207-210.

De los Santos, PT2011,'Caracterización fenotípica de las poblaciones de cabras lecheras en el valle de Libres, estado de Puebla'Tesis de maestría, Colegio de Postgraduados.

Dorantes, CEJ, Torres, HG, Castañeda, BVJ, Hernández, MO, Gallegos, SJ, Becerril, PCM \& Rojo, RR 2012, 'Limitantes socioeconómicas de los sistemas de producción caprina en el sur del estado de México',Actas Iberoamericanas de Conservación Animal,vol.2, pp. 333-36.

Escareño, SLM, Wurzinger, M, Pastor, LF,Salinas, H, Sölkner, J \&lñiguez, L2011, 'La cabra y los sistemas de producción caprina de los pequeños productores de la Comarca Lagunera, en el norte de México', Revista Chapingo. Serie Ciencias Forestales y del Ambiente,vol.17. pp. 235-246.

Gaspar, P, Escribano, M, Pulido, F, Rodríguez-Ledesma, A, Mesías, FJ \& Pulido, AF 2016, 'El papel de la gestión cooperativa en la mejora del funcionamiento técnico-económicode explotaciones ovinas', Archivos de Zootecnia, vol. 65, pp. 333-39.

Gómez, NC, Bustinza, RH, Revidatti, MA, Ferrando, A, Milán. MJ \&Jordana, J2012,'Caracterización morfológica y faneróptica de la cabra Apurimeña Peruana', Actas Iberoamericanas de Conservación Animal, vol. 2, pp. 57-60.

Guerrero, LM, Téllez, JN, Vera, M, Garza, BA, Rincón, JL,Martínez, BD, Serrano, NC \& Vargas,BJE2012, 'Caracterización de los sistemas de producción caprinos presentes en el municipio de Jordán, Santander, Colombia',Actas Iberoamericanas de Conservación Animal, vol. 2, pp. 171-74. 
Gül, M, Demircan, V, Yilmaz, H \& Yilmaz H 2016, 'Technical efficiency of goat farming in Turkey: a case study of Ispartaprovince', RevistaBrasileira de Zootecnia, vol. 45, pp. 328-35.

Gunia, M, Mandonnet N, Arquet, R, De la Chevrotiére, C, Naves, M, Mahieu, M \&Alexandre, G2010, 'Production systems of Creole goat and their implications for a breeding programme', Animal,vol. 4, pp. 2099-2105.

Hernández, ZJS, Franco, GFJ, Herrera, GM, Rodero, SE, Sierra, VAC, Bañuelos, CA \&Delgado, BJV2002, 'Estudio de los recursos genéticos de México: características morfológicas y morfoestructurales de los caprinos nativos de México',Archivos de Zootecnia, vol. 51, pp. 53-64.

Hernández, ZJS, Pérez, AR, Ramírez, HR, Rivera, AG \&Reséndiz, MR 2012,'Estrategias de manejo del recurso genético caprino en Puebla, México',Actas Iberoamericanas de Conservación Animal, vol. 2, pp. 143-46.

Herrera, M\& Luque, M2009,'Valoración morfológica en el ganado caprino extensivo de carne', en AC Sañudo (comp.), Valoración Morfológica de los Animales Domésticos, Ministerio de Medio Ambiente y Medio Rural y Marino, Madrid, España.

Hulela, K 2010, 'The role of women in sheep and goats production in Sub-Saharan Africa ', International Journal of Scientific Research in Education, vol. 3, pp. 177-187.

INEGI, Instituto Nacional de Estadística Geografía e Informática2012,Anuario Estadístico de Colima2012, visto 10 Julio 2105, http://www.inegi.org.mx/est/contenidos/espanol/sistemas/ aee $12 /$ estatal/col/default.htm.

Luque, CM2011, 'Caracterización y evaluación de las razas caprinas autóctonas españolas de orientación cárnica' Tesis de doctorado, Universidad de Córdoba.

Luque M, Pérez-Sempere, I, Sánchez, MD, García, A, Rodero, E \&Herrera M 2005, 'Análisis comparativo de los caracteres cualitativos externos en las razas Blanca Celtibérica y Blanca Andaluza' Memorias de las XXX Jornadas Científicas de la Sociedad Española de Ovinotecnia y Caprinotecnia, Sociedad Española de Ovinotecnia y Caprinotecnia, Granada, España, pp. 107-110.

Martínez, RRD, Torres, HG \&Martínez, HS2013,'Caracterización fenotípica, productiva y reproductiva de la cabra blanca Criolla del "Filo Mayor" de la Sierra Madre del Sur en el estado de Guerrero', Nova Scientia, vol. 6, pp.25-44.

Martínez-Rojero RD, Torres-Hernández, G, Mastache-Lagunas, AA, Rubio-Rubio, M, Sánchez de la Rosa, I, González-Acevedo, H \& Rodríguez-Adán, H2004, 'Caracterización de un rebaño caprino criollo Celtibérico en el trópico seco del estado de Guerrero', Memorias de la XIX Reunión Nacional sobre Caprinocultura, Asociación Mexicana de Profesionistas en Caprinos, Acapulco, México, pp. 232-236.

Mellado, M1997,'La cabra criolla en América Latina', Veterinaria México, vol. 28, pp. 333-343.

Ministerio de Agricultura y Pesca, Alimentación y Medio Ambiente 2017 Raza caprina Blanca Celtibérica, vistoel 25 de Abril de 2017,http:// www.mapama.gob.es/es/ganaderia/temas/zootecnia/razas-ganaderas/razas/catalogo/peligro-extincion/caprino/blanca-celtiberica/

Montaldo, HH, Torres-Hernández, G \& and Valencia-Posadas, M2010,'Goat breeding research in Mexico', Small Ruminant Research,vol. 89, pp. 155-163.

Morand-Fehr, P, Boutonnet, JP, Devendra, C, Dubeuf, JP,Haenlein, JFW, Holst, P,Mowlem, L \&Capote, J2004, 'Strategy for goat farming in the 21 st century', Small Ruminant Research, vol. 51, pp. 175-183.

Nozawa, K1991,'Domestication and history of goats', in E Maijala (ed.), Genetics resources of pig, sheep and goat, Elsevier Science Publishers, Amsterdam, Netherlands.

Oliveira Moura, J, Guisares-Campelo, JE, Bajay, MM, Lindenberg Rocha Sarmento, J, Barros-Britto, F, Leal, TM, Silva-Costa, M, HelciasCavalcante, D, Figueiredo-Carvalho, MD, Mello-Araújo, A 2019 'Diversidade genética em caprino Marota e manejo genético para rebanhos de conservação', Archivos de Zootecnia, vol.68, pp. 138-145.

Pardos L, Maza, MT, Fantova, E \&Sepúlveda, W2008, 'The diversity of sheep production systems in Aragón (Spain): characterisation and typification of meat sheep farms', Spanish Journal of Agricultural Research, vol. 6, no. 4, pp. 497-507.

Ramírez, JMP, Sánchez, OM,Ortiz, BR, Zaragoza, RJL,Ricardi, DLC \&Fuentes, MG, 2014, 'Sistema de producción y zoometría de la cabra Pastoreña de la Mixteca oaxaqueña',Actas Iberoamericanas de Conservación Animal, vol. 4, pp. 231-33.

Reveles, TLR, Echavarría, HF, Bañuelos, VR, Salinas, GH \&Cabral, AFJ2008, 'Empleo de marcadores moleculares en la diferenciación de razas caprinas del Estado de Zacatecas, México', Tropical and Subtropical Agroecosystems,vol. 9, pp. 15-27.

Revidatti, MA, Prieto, PN, De La Rosa, S, Ribeiro, MN \&Capellari, A2007,'Cabras criollas de la región norte Argentina. Estudio de variables e índices zoométricos',Archivos de Zootecnia, vol. 56, pp. 479-482.

Ricordeau, G1967, 'Hérédité des pendeloques en race Saanen. Différence de fécondité entre les génotypes avec et sans pendeloques', Annales de Zootechnie, vol.16, pp. 263-270.

Rodríguez, PL, Tovar, JJ, Rota, AM, Rojas, A \&Martín, L1990,'El exterior de la cabra Verata', Archivos de Zootecnia, vol. 39, pp. 43-57.

Rossanigo, CE, Frigerio, KL, Silva, CJ \& Boza J1996,'Resultados de producción de la cabra criolla sanluiseña (Argentina)',Memoria de las XXI Jornadas Científicas de la Sociedad Española de Ovinotecnia y Caprinotecnia, Sociedad Española de Ovinotecnia y Caprinotecnia, Logroño, España, pp. 259-266.

Ruiz, F, López, H, Salinas, L, Aguirre, JJG \& Suarez EJ1992,'Goat production characterization in southeast of Coahuila in northern México', Proceedings of the 5th Conference on Goats, International Goat Association, New Dheli, India, pp. 434-438.

Ruiz, LI2008,Valoración morfológica en ganado caprino lechero. Cabra Murciano- Granadina, Comunidad Autónoma de la Región de Murcia, Consejería de Agricultura y Agua, Murcia, España.

Sánchez-Gutiérrez, R, Gutiérrez-Luna, R \& Flores-Nájera, MJ 2018, 'Caracterización morfológica de un rebaño de conservación de cabras criollas en Zacatecas, México', Archivos de Zootecnia, vol.67, pp. 73-79.

Sánchez, RM, Muñoz, MME, Cárdenas, BJM\&Blanco, CG2009, 'Valoración morfológica en el ganado caprino extensivo de carne', en AC Sañudo (comp.), Valoración Morfológica de los Animales Domésticos, Ministerio de Medio Ambiente y Medio Rural y Marino, Madrid, España.

Scheaffer RL, Mendenhall, W \&OH, L1990, Elementary Survey Sampling, 4 th edn, PWS-Kent, Boston, USA

Sedano, TDA2012, 'La cabra Pastoreña para la producción de carne en el municipio de Tlalixtaquilla, Guerrero'Tesis de maestría, Colegio de Postgraduados.

Serrano, OML2010,'Análisis del sistema de producción de cabras con fines lecheros en la región de Libres, Puebla' Tesis de maestría, Colegio de Postgraduados.

SIIC, Sistema Integral de Información Colima 2007. Mapa regiones fisiográficas, Universidad de Colima, Dirección General de Estudios Estratégicos, visto 25 Enero 2015, http://siic.ucol.mx/.

Silva-Gómez, S, Rodríguez, G, Zaragoza, L, Pérez, R \&Toxtle, J2015,'Caracterización de hatos caprinos en una localidad mexicana',Actas Iberoamericanas de Conservación Animal, vol.6, pp. 226-233.

SPSS 2006, SPSS Base 15.0 user's guide, International Business Machines Corporation, Chicago, Illinois, USA.

Trujillo OCX2012,'El cruzamiento de la cabra criolla para carne con razas mejoradas en la Mixteca Poblana, México' Tesis de maestría, Colegio de Postgraduados.

Valencia, M\&Montaldo, HH 2006, 'Genetic evaluation of goats in the state of Guanajuato, Mexico', Proceedings of the 8th World Congress on Genetics Applied to Livestock Production, InstitutoProciência, Belo Horizonte, Brazil, pp. 2-6 
Valerio, D, García, A, Perea, J, Acero, R\& Gómez, G2009,'Caracterización social y comercial de los sistemas ovinos y caprinos de la región noroeste de Republica Dominicana', Interciencia, vol. 34, pp. 337-644.

Vargas, BJE, Serrano, NCA, Martínez, BDA, Rodríguez, G \& Zaragoza, L 2014, 'Los sistemas productivos caprinos en la provincia de Guanentá, Santander, sobre la cuenca del río Chicamocha', Actas Iberoamericanas de Conservación Animal, vol. 4, pp. 237-39.
Vargas, SL2003,'Análisis y desarrollo del sistema de producción agrosilvopastoril caprino para carne en condiciones de subsistencia en Puebla, México' Tesis de doctorado, Universidad de Córdoba.

Vázquez-García, V 2015, 'Ganado menory enfoque de género. Aportes teóricos y metodológicos', Agricultura, Sociedad y Desarrollo, vol. 12, pp. 515-531. 